\title{
étude probabiliste du comportement d'un remblai sur sols mous
}

\author{
par \\ J.-P. Magnan S. Baghery \\ Laboratoire Central des Ponts et Chaussées, Paris
}

\section{Introduction}

Malgré le grand développement des recherches sur ranalyse probabiliste du comportement des ouvrages au cours des dix dernières années, la prédiction probabiliste des amplitudes ou vitesses de déformation des sols de fondation compressibles a été peu traitée dans la littérature internationale : six articles seulement s'intéressent à ce type de problèmes alors qu'il y en a, par exemple, près de 25 pour les études de stabilité. Le premier (Folayan et al., 1970) ne concernait d'ailleurs pas vraiment la prédiction des tassements en elle-même mais plutôt la façon d'organiser la reconnaissance géotechnique en s'appuyant sur la théorie de la décision. Par la suite, Vanmarcke et Fuleihan (1975) ont proposé une méthode analytique pour prédire la densité de probabilité du tassement final. calculé par la méthode œdométrique; Freeze (1977) a utilisé la méthode de Monte Carlo pour prédire le degré de consolidation unidimensionnel; Asaoka (1978) et Asaoka et Suzuki (1979) ont proposé une méthode analytique pour extrapoler les courbes de tassement observées; Chang et Soong (1979) ont développé une solution analytique pour calculer la valeur moyenne et l'écart-type du degré de consolidation de la théorie de la consolidation unidimensionnelle de Terzaghi.

C'est en 1978 qu'a été entreprise au Laboratoire Central des Ponts et Chaussées une étude systématique des méthodes d'analyse probabiliste du comportement des ouvrages, du point de vue de la mécanique des sols. Cette étude a été orientée vers une classe de problèmes pour laquelle existaient de nombreuses données expérimentales: les remblais sur sols compressibles et plus particulièrement ceux du site expérimental de Cubzac-les-Ponts. Le comportement du remblai $B$, objet du présent article, a été analysé en deux temps: on a d'abord caractérisé d'un point de vue statistique les propriétés physiques et mécaniques du sol de fondation; puis on a calculé le tassement final, le degré de consolidation et le tassement au cours du temps par différentes méthodes probabilistes.
Ces résultats ont été ensuite comparés à ceux des mesures effectuées sur le site pendant les trois années suivant la construction du remblai.

\section{Le cas traité : remblai B du site expérimental de Cubzac-les-Ponts}

Le site expérimental de remblais sur sols compressibles des Laboratoires des Ponts et Chaussées à Cubzac-les-Ponts (Gironde) a déjà fait l'objet de différentes publications (Vogien, 1975; Blondeau et al., 1977; Dang et Magnan, 1977; Magnan et al.. 1978; Belkeziz et Magnan, 1982; Shahanguian, 1980), dont l'une était justement consacrée à l'objet de la présente étude, le remblai $B$ (hauteur $2,3 \mathrm{~m}$ ), édifié en 1975 avec un coefficient de sécurité de 1.5 et destiné à l'étude de la consolidation du sol de fondation sous le remblai et dans son voisinage (Magnan et al., 1978).

Sur le site, les alluvions compressibles sont constituées du haut vers le bas par:

- une mince couche de terre végétale, d'une épaisseur approximative de $0,30 \mathrm{~m}$;

- une couche d'argile limoneuse d'une épaisseur de 1 à $2 \mathrm{~m}$, formant une croûte surconsolidée et altérée:

- une couche d'argile molle grise plus ou moins organique, dont l'épaisseur atteint $8 \mathrm{~m}$.

Le substratum est constitué d'une couche de graves située vers $10 \mathrm{~m}$ de profondeur, d'épaisseur moyenne $5 \mathrm{~m}$, reposant sur des roches marneuses ou calcaires.

L'argile du site présente une bonne homogénéité. Néanmoins, on observe des litages de fibres végétales bien conservées vers 2 à $3 \mathrm{~m}$ de profondeur. La cote NGF au niveau du sol est de $+2 \mathrm{~m}$. Le toit de la nappe fluctue chaque année entre la surface du terrain - naturel et $1,5 \mathrm{~m}$ de profondeur.

Les données géotechniques disponibles pour la zone du remblai B proviennent de 4 sondages carottés (au carottier à piston stationnaire) ayant fourni le sol pour 
les essais de laboratoire, 11 sondages pénétrométriques, 5 sondages scissométriques et 6 sondages pressiométriques. La figure 1 montre l'emplacement des sondages carottés et des essais en place par rapport au remblai B.

L'analyse statistique des valeurs des paramètres géotechniques du sol de fondation du remblai B a conduit aux résultats représentés sur la figure 2 et dans le tableau 1 pour les paramètres de compressibilité (indice des vides initial $e_{0}$, poids volumique $\gamma$, indice de compression $\mathrm{C}_{\mathrm{c}}$, indice de gonflement $\mathrm{C}_{\mathrm{s}}$ et pression de préconsolidation $\alpha_{p}^{\prime}$ ). Pour le coefficient de consolidation $c_{v}$ du sol normalement consolidé, le nombre des valeurs disponibles était inférieur à 10 et l'on a admis que $c_{v}$ suit une loi log-normale, les valeurs de $c_{v}$ étant supposées constantes au cours du temps (valeur moyenne $\overline{\mathrm{c}}_{\mathrm{v}}=2,1 \cdot 10^{-8} \mathrm{~m}^{2} / \mathrm{s}$; coefficient de variation $\mathrm{CV}\left[\mathrm{c}_{\mathrm{v}}\right]=0,76$ ).

Pour certains calculs, on a besoin des valeurs des coefficients de corrélation entre les paramètres, pris deux à deux. Ces coefficients de corrélation sont rassemblés dans le tableau 2.

Enfin, les variations spatiales des paramètres $e_{0}, \gamma, C_{c}$ et $\sigma_{\mathrm{p}}^{\prime}$ sur l'épaisseur de la couche compressible ont èté analysées à l'aide d'un modèle de régression linéaire à l'intérieur de trois couches de $1,5 \mathrm{~m}-2 \mathrm{~m}$ et $5,5 \mathrm{~m}$. respectivement (fig. 3).

\subsection{Calcul des amplitudes des tassements}

Deux méthodes de calcul ont été utilisées pour calculer le tassement final du sol sous le remblai B : la méthode de Monte Carlo et une méthode analytique utilisée par Vanmarcké et Fuleihan (1975).

Dans les deux cas, on a considéré la charge appliquée par le poids du remblai à la surface du sol comme un paramètre déterministe $(48,3 \mathrm{kPa})$. La distribution des surcontraintes induites par cette charge dans la couche compressible a été déterminée à l'aide de l'abaque d'Osterberg (charge en forme de demiremblai).

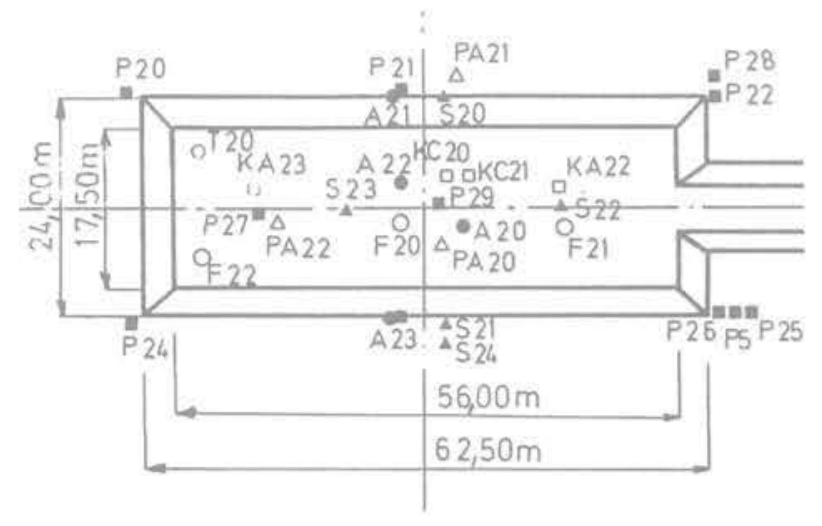

$$
\begin{aligned}
& \text { - Sondage carotté toSondage tariere } \\
& \text { - Scissometre ospressiometre } \\
& \text { - Pénétromètre assai de perméabilite } \\
& \text { en place }
\end{aligned}
$$

Fig. 1 Emplacement des essais en place et des sondages carottés (Remblai B de Cubzac-les-Ponts)
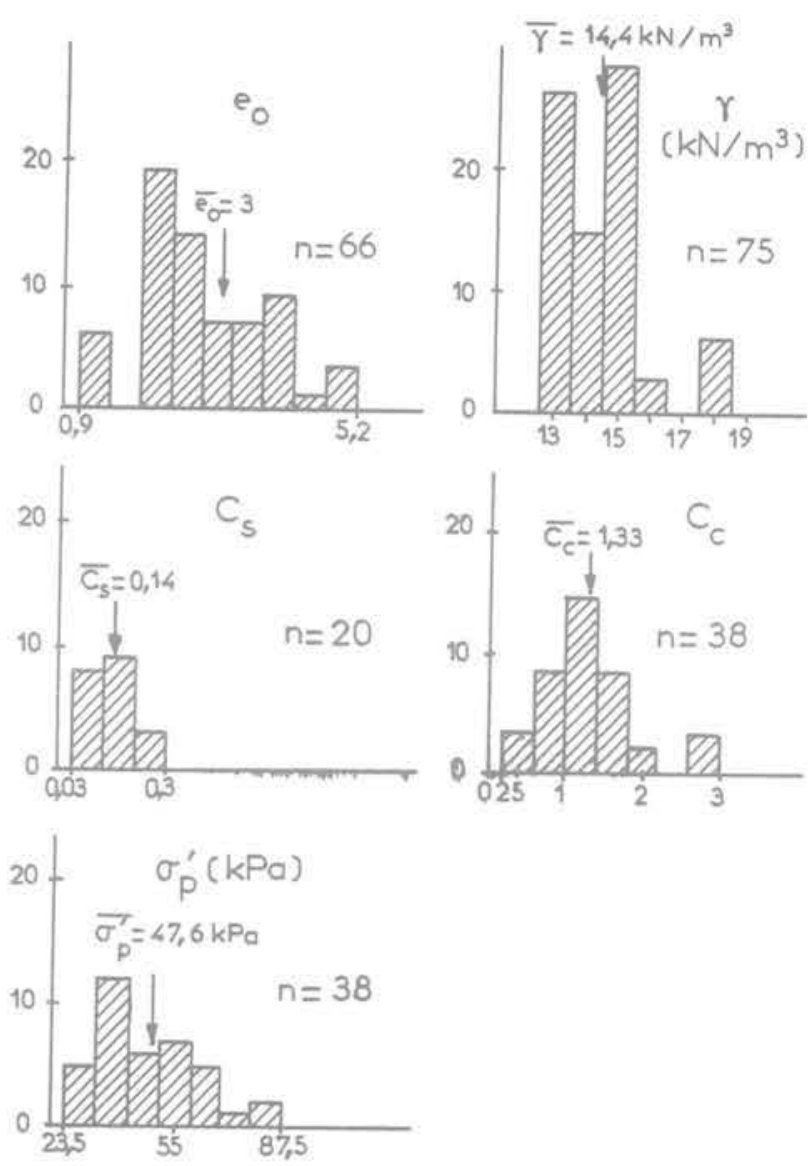

Fig. 2

Tableau 1

\begin{tabular}{|c|c|c|c|c|c|}
\hline \multirow[b]{2}{*}{ Couche } & \multicolumn{5}{|c|}{ Coefficient de variation/Loi adoptée } \\
\hline & $e_{0}$ & $\gamma$ & $\mathrm{c}_{\mathrm{s}}$ & $\mathrm{C}_{\mathrm{c}}$ & $\sigma_{p}^{\prime}$ \\
\hline 0 à $1 \mathrm{~m}$ & $0,09_{\mathrm{N}}$ & $0,022_{N}$ & - & - & $0,14_{\mathrm{N}}$ \\
\hline 1 à $2 \mathrm{~m}$ & $0,19_{\mathrm{N}}$ & $0,07 \mathrm{~N}$ & - & - & - \\
\hline 2 à $3 \mathrm{~m}$ & $0,15_{N}$ & $0,02_{\beta}$ & - & $0,06_{N}$ & $0,18_{N}$ \\
\hline 3 à $4 \mathrm{~m}$ & $0,21_{N}$ & $0,07_{\beta}$ & - & $0,28_{\mathrm{N}}$ & $0,16_{\beta}$ \\
\hline 4 à $5 \mathrm{~m}$ & $0,11_{N}$ & $0,02_{\beta}$ & - & $0,26_{N}$ & $0,09_{\beta}$ \\
\hline 5 à $6 \mathrm{~m}$ & $0,09_{N}$ & $0,04_{\beta}$ & - & $0,13_{N}$ & $0,17_{\beta}$ \\
\hline 6 à $7 \mathrm{~m}$ & $0,05_{N}$ & $0,02_{\beta}$ & - & $0,15_{N}$ & $0,22_{\beta}$ \\
\hline 7 à $8 \mathrm{~m}$ & $0,07_{N}$ & $0,05_{\beta}$ & - & $0,18_{N}$ & $0,21_{\beta}$ \\
\hline 8 à $9 \mathrm{~m}$ & $0.03_{\mathrm{N}}$ & $0,02_{\beta}$ & - & $0,07_{N}$ & $0,06_{\beta}$ \\
\hline \multicolumn{3}{|c|}{$\mathrm{N}$ - Loi normale } & \multicolumn{3}{|c|}{$\beta$ - Loi bêta } \\
\hline
\end{tabular}

Coefficient de variation et loi de probabilité des valeurs des paramètres de compressibilité (remblai $B$ de Cubzac-les-Ponts) 
Tableau 2

Coefficients de corrélation entre les paramètres utilisés dans les calculs de tassement

(Remblai B de Cubzac-les-Ponts)

\begin{tabular}{|c|cccccc|}
\hline & $\mathrm{e}_{\mathrm{o}}$ & $\sigma_{\mathrm{p}}^{\prime}$ & \multicolumn{1}{c}{$\mathrm{C}_{\mathrm{c}}$} & \multicolumn{1}{c|}{$\mathrm{C}_{\mathrm{v}}$} & \multicolumn{1}{c|}{$\sigma_{\mathrm{vo}}^{\prime}$} & \multicolumn{1}{c|}{$\mathrm{C}_{\mathrm{s}}$} \\
\hline $\mathrm{e}_{\mathrm{o}}$ & 1 & $-0,44$ & 0,47 & $-0,39$ & $-0,14$ & 0,38 \\
$\sigma_{\mathrm{p}}^{\prime}$ & & 1 & $-0,15$ & 0,58 & 0,32 & $-0,53$ \\
$\mathrm{C}_{\mathrm{c}}$ & & & 1 & $-0,80$ & $-0,11$ & 0,70 \\
$\mathrm{C}_{\mathrm{v}}$ & & & & 1 & $-0,49$ & $-0,79$ \\
$\sigma_{\mathrm{v}}^{\prime}$ & & & & & 1 & $-0,14$ \\
$\mathrm{C}_{\mathrm{s}}$ & & & & & & 1 \\
\hline
\end{tabular}

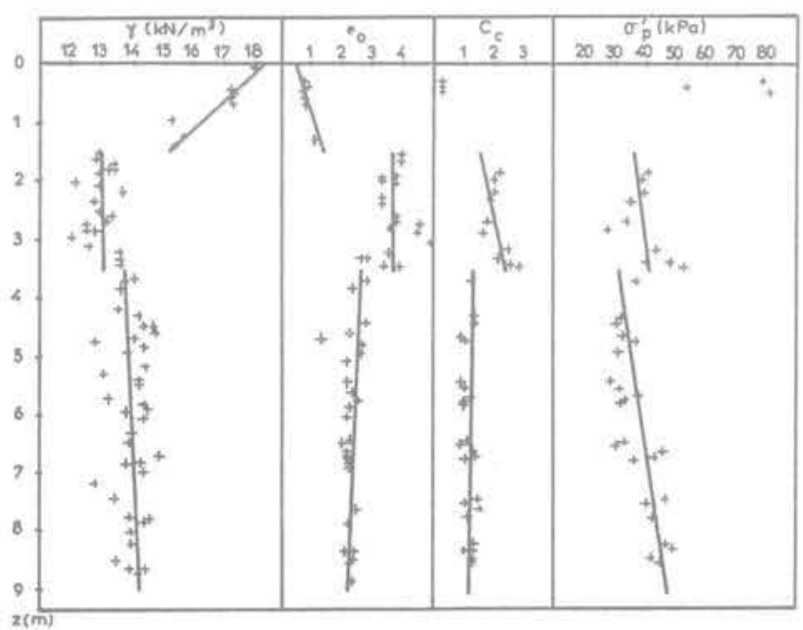

Fig. 3 Variations de $\gamma, e_{0}, C_{C}$ et $\sigma$ 'p sur l'épaisseur de la couche compressible (Cubzac - B)

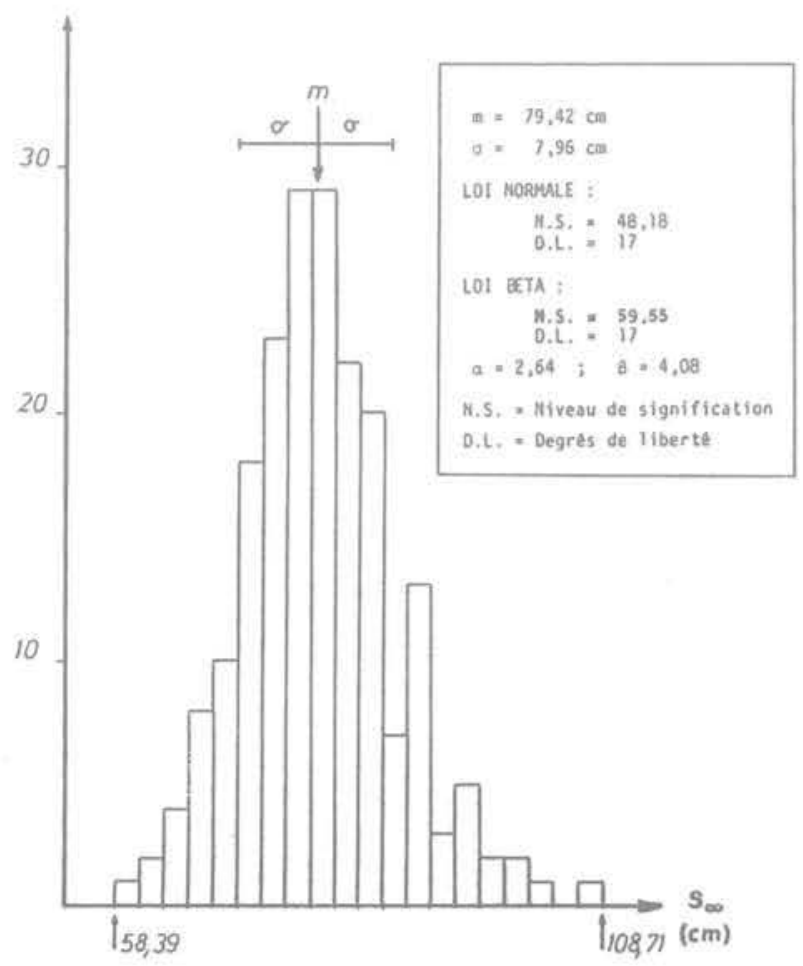

Fig. 4 Histogramme et coefficients statistiques du tassement final

\section{Méthode de Monte-Carlo}

Cette méthode consiste à calculer un grand nombre de fois le tassement final du sol en attribuant aux paramètres de la formule de calcul des valeurs "tirées" au hasard d'après les lois de distribution observées pour ces paramètres puis à effectuer une analyse statistique des résultats de ces calculs.

Dans le cas de Cubzac, 9 couches de sol ont été distinguées et le tassement final de la couche compressible a été pris égal à la somme des tassements finaux de chacune de ces neuf couches :

$$
\mathrm{s}_{\infty}=\sum_{i} \mathrm{~h}_{\mathrm{oi}}\left[\frac{\mathrm{C}_{\mathrm{ci}}}{1+\mathrm{e}_{\mathrm{oi}}} \lg \frac{\sigma_{\mathrm{voi}}^{\prime}+\Delta \sigma_{\mathrm{vi}}}{\sigma_{\mathrm{pi}}^{\prime}}+\frac{\mathrm{C}_{\mathrm{si}}}{1+\mathrm{e}_{\mathrm{oi}}} \times \lg \frac{\sigma_{\mathrm{pi}}^{\prime}}{\sigma_{\mathrm{voi}}^{\prime}}\right]
$$

avec les notations suivantes:

$e_{o} \quad$ indice des vides initial de la $i$-ème couche,

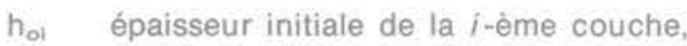

$\mathrm{C}_{\mathrm{si}}$ indice de gonflement de la $i$-ème couche,

$\mathrm{C}_{\mathrm{c}}$ indice de compression de la $i$-ème couche,

$\sigma_{\text {voi }}^{\prime}=\sum_{j=1}^{i-1} \gamma_{i} h_{0 i}+\frac{1}{2} \gamma_{i} h_{0 i}-u_{i}$

contrainte effective verticale initiale au milieu de la $i$-ème couche,

$\gamma_{i}$ poids volumique du sol dans la $i$-ème couche,

$u_{i}$ pression interstitielle au milieu de la $i$-ème couche (on a supposé la nappe phréatique à $-1 \mathrm{~m}$ de profondeur).

$\Delta \sigma_{\mathrm{vi}} \quad$ Surcontrainte verticale induite par le poids du remblai au milieu de la $i$-ème couche,

$\sigma_{\mathrm{p} i}^{\prime} \quad$ pression de préconsolidation au milieu de la $i$-ème couche.

Les paramètres des 200 calculs du tassement final ont été générés de façon aléatoire en tenant compte de la dérive linéaire en fonction de la profondeur dans tous les cas oủ elle était significative, en utilisant les lois de distribution et les coefficients statistiques estimés dans chaque couche.

Dans chaque couche, compte tenu de la loi de distribution, des coefficients statistiques et de la dérive linéaire des paramètres, les valeurs de ceux-ci sont "tirées" à chaque itération du calcul, le tassement final de la $i$-ème itération étant égal à la somme des tassements finaux des 9 couches pour cette itération. Les 200 valeurs du tassement final ainsi obtenues ont permis de déterminer la valeur moyenne, l'écart-type, les valeurs extrêmes et les paramètres de la loi de distribution du tassement final (dans les cas d'une loi normale et d'une loi bêta). La fonction de répartition du tassement final est représentée sous forme d'histogramme sur la figure 4.

\section{Méthode analytique}

Cette méthode repose sur le développement en série de Taylor de la formule de calcul des tassements de la couche $\mathrm{i}$ :

où :

$$
\begin{aligned}
& \mathrm{s}=\mathrm{h} \cdot \frac{\mathrm{C}_{\mathrm{x}}}{1+\mathrm{e}_{\mathrm{o}}} \cdot \lg \frac{\sigma_{1}}{\sigma_{2}}=\mathrm{h} \cdot \mathrm{C}_{\mathrm{A}} \cdot \mathrm{L} \\
& \mathrm{C}_{\mathrm{A}}=\frac{\mathrm{C}_{\mathrm{x}}}{1+\mathrm{e}_{\mathrm{o}}} \text { et } \mathrm{L}=\lg \frac{\sigma_{1}}{\sigma_{2}} .
\end{aligned}
$$

D'après Benjamin et Cornell (1970), on peut utiliser les relations suivantes pour calculer la moyenne $E\left[s_{i}\right]$ et le 
coefficient de variation $\mathrm{CV}\left[\mathrm{s}_{\mathrm{i}}\right]$ du tassement de la couche $\mathrm{i}$ :

$E[s]=h \cdot E\left[C_{R}\right] \cdot E[L]$,

$\mathrm{CV}^{2}[\mathrm{~s}]=\mathrm{CV}^{2}\left[\mathrm{C}_{\mathrm{F}}\right]+\mathrm{CV}^{2}[\mathrm{~L}]+\mathrm{CV}\left[\mathrm{C}_{\mathrm{R}}\right] \cdot \mathrm{CV}[\mathrm{L}]$

$\approx C V^{2}\left[C_{A}\right]+C V^{2}[L]$.

On peut montrer, dans l'hypothèse oủ les coefficients de variation de $C_{x}$ (égal à $C_{s}$ ou $C_{c}$ selon que le sol est surconsolidé ou normalement consolidé) et de $1+e_{0}$ sont petits, que les termes précédents sont égaux à :

$$
\begin{aligned}
& E\left[C_{F}\right] \simeq \frac{\bar{C}_{x}}{1+\bar{e}_{0}} \\
& E[L]=\lg \frac{E\left[\sigma_{1}\right]}{E\left[\sigma_{2}\right]}-0,22\left(\operatorname{CV}^{2}\left[\sigma_{1}\right]+\operatorname{CV}^{2}\left[\sigma_{2}\right]\right) \text {, } \\
& \mathrm{CV}\left[\mathrm{C}_{\mathrm{F}}\right]=\sqrt{\mathrm{CV}^{2}\left[\mathrm{C}_{\mathrm{x}}\right]+\mathrm{CV}^{2}\left[1+\mathrm{e}_{0}\right]} \text {, } \\
& \mathrm{CV}[\mathrm{L}]=0,434 \sqrt{\frac{\mathrm{CV}^{2}\left[\sigma_{1}\right]+\mathrm{CV}^{2}\left[\sigma_{2}\right]}{E(L)}} .
\end{aligned}
$$

Sur cette base, on peut calculer le tassement final de chacune des neuf couches et le tassement final du sol

$$
\mathbf{S}_{\infty}=\sum_{i=1}^{9} \mathbf{S}_{i} \text {. }
$$

La moyenne et la variance de $\mathrm{s}_{\infty}$ sont données par les expressions :

$$
\begin{aligned}
& E\left[S_{\infty}\right]=\sum_{i=1}^{9} E\left[s_{i}\right] \\
& V\left[S_{\infty}\right]=\sum_{i=1}^{9} V\left[s_{i}\right]+2 \sum_{i=1}^{9} \sum_{j>i} \rho_{i j} \sigma_{s_{i}} \sigma_{s_{i}}
\end{aligned}
$$

où les $\rho_{i j}$ sont les coefficients de corrélation des tassements des couches $i$ et $\mathrm{j}$ et les $\sigma_{\mathrm{s}}$ et $\sigma_{\mathrm{s}}$ les écarts-types de ces tassements. Faute de disposer de données fiables sur les valeurs des $\rho_{i j}$, on a fait le calcul dans le cas où tous les $\rho_{i,}$ sont nuls, c'est-à-dire où les tassements des neuf couches du sol sont indépendants les uns des autres.

Le calcul donne pour la valeur moyenne et l'écart-type du tassement final les valeurs suivantes:

$$
\overline{\mathrm{s}}_{\infty}=80 \mathrm{~cm} \quad \sigma_{\mathrm{s}_{\alpha}}=11,8 \mathrm{~cm} \text {. }
$$

Par comparaison, la méthode de Monte-Carlo conduit à

$$
\overline{\mathrm{s}}_{\infty}=79,4 \mathrm{~cm} \quad \sigma_{\mathrm{s}_{\infty}}=8 \mathrm{~cm},
$$

tandis qu'un calcul déterministe effectué avec les valeurs moyennes des paramètres de chaque couche donne $\mathrm{s}_{\infty}=80 \mathrm{~cm}$.

\subsection{Calcul des vitesses de consolidation}

Cette partie de l'étude a également été réalisée par deux méthodes : la méthode de Monte Carlo et la méthode analytique de Chang et Soong (1979).

\section{Méthode de Monte-Carlo}

Pour appliquer la méthode de Monte-Carlo, on a utilisé le programme de calcul CONMULT (Consolidation unidimensionnelle des multicouches, THOMANN 1972) pour le calcul de la vitesse de consolidation, en considérant comme seule variable aléatoire le coefficient de consolidation $\mathrm{c}_{\mathrm{v}}$. Ce programme résout par la méthode des différences finies l'équation de la consolidation unidimensionnelle. II faut introduire comme données la discrétisation spatiale du sol (nombre de sous-couches) et la valeur des paramètres du calcul dans chaque couche. Pour le calcul en différences finies, on a choisi de diviser le sol de fondation en 50 sous-couches, regroupées en 9 couches de $1 \mathrm{~m}$ d'épaisseur. Cent calcùls ont été effectués, avec des valeurs aléatoires du coefficient de consolidation dans chaque couche. Dans chaque calcul on a supposé que le coefficient de perméabilité du sol était proportionnel au coefficient de consolidation correspondant, c'est-à-dire que la condition de continuité de l'écoulement entre les couches pouvait s'exprimer en fonction de

$$
\mathrm{k}_{\mathrm{vi}} / \mathrm{k}_{\mathrm{vi}+1}=\mathrm{c}_{\mathrm{vi}} / \mathrm{c}_{\mathrm{vi}+1} \text {. }
$$

Pour la génération des 900 valeurs nécessaires du coefficient de consolidation, on a utilisé une loi de distribution log-normale, choisie après application du text de Shapiro-Wilk. Les valeurs obtenues ont été supposées constantes au cours du temps.

Les résultats du calcul sont représentés sur la figure 5 sous forme d'histogrammes des temps nécessaires pour atteindre une valeur donnée du degré de consolidation et d'une courbe reliant les valeurs moyennes du temps au bout duquel on atteint un degré de consolidation de U.

Pour chaque degré de consolidation on a testé trois lois (normale, log-normale, bêta); le tableau 3 présente les coefficients statistiques des temps de consolidation calculés et les niveaux de signification des lois testées.

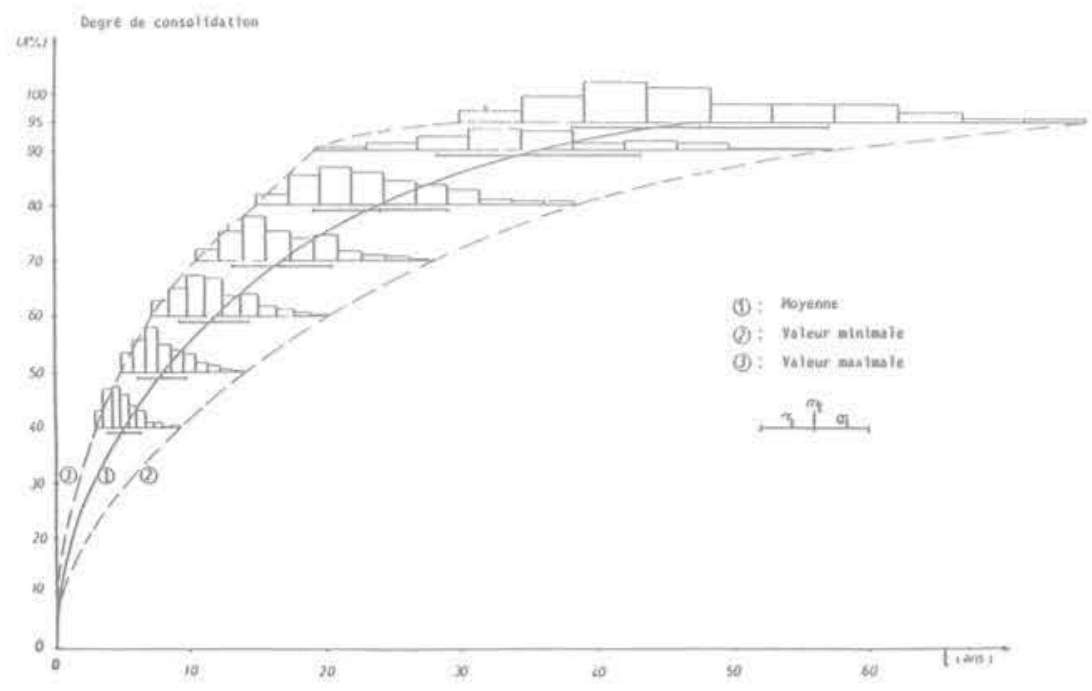

Fig. 5 Evolution du degré de consolidation du sol au cours du temps 
Tableau 3

Moyenne et écart-type du temps nécessaire pour atteindre différentes valeurs du degré de consolidation et niveaux de signification des lois testées

\begin{tabular}{|c|c|c|c|c|c|c|}
\hline $\begin{array}{l}\text { Temps } \\
\mathrm{U} \%\end{array}$ & $\begin{array}{l}\text { Moyenne } \\
\text { (années) }\end{array}$ & $\begin{array}{c}\text { Écart-Type } \\
\text { (années) }\end{array}$ & $\begin{array}{c}\text { N.S. de la } \\
\text { loi normale } \\
(\%)\end{array}$ & $\begin{array}{c}\text { N.S. de la } \\
\text { loi log-normale } \\
(\%)\end{array}$ & \multicolumn{2}{|c|}{$\begin{array}{c}\text { N.S. de la } \\
\text { loi bêta et } \\
\text { valeurs de } \alpha \text { et } \beta\end{array}$} \\
\hline 10 & 0,31 & 0,09 & 3,44 & 0,02 & 6,18 & $\begin{array}{l}\alpha=0,19 \\
\beta=1,72\end{array}$ \\
\hline 20 & 1,23 & 0,36 & 3,11 & 17,66 & 24,02 & $\begin{array}{l}\alpha=0,38 \\
\beta=2,01\end{array}$ \\
\hline 30 & 2,83 & 0,76 & 4,20 & 34,08 & 22,29 & $\begin{array}{l}\alpha=0,31 \\
\beta=1,88\end{array}$ \\
\hline 40 & 5,13 & 1,26 & 24,02 & 93,74 & 78,58 & $\begin{array}{l}\alpha=0,43 \\
\beta=1,96\end{array}$ \\
\hline 50 & 8,12 & 1,87 & 25,85 & 89,17 & 10.68 & $\begin{array}{l}\alpha=0,54 \\
\beta=1,95\end{array}$ \\
\hline 60 & 11,90 & 2,63 & 29,78 & 27,77 & 57,14 & $\begin{array}{l}\alpha=0,58 \\
\beta=1,87\end{array}$ \\
\hline 70 & 16,80 & 3,54 & 24,02 & 19,11 & 73,54 & $\begin{array}{l}\alpha=0,68 \\
\beta=1,95\end{array}$ \\
\hline 80 & 23,82 & 4,97 & 31,89 & 36,37 & 54,38 & $\begin{array}{l}\alpha=0,65 \\
\beta=1,77\end{array}$ \\
\hline 90 & 35,72 & 7,49 & 15,01 & 36,37 & 85,34 & $\begin{array}{l}\alpha=1,30 \\
\beta=2,06\end{array}$ \\
\hline 95 & 47,74 & 9,58 & 15,01 & 4,63 & 89,17 & $\begin{array}{l}\alpha=0,72 \\
\beta=1,77\end{array}$ \\
\hline
\end{tabular}

\section{Méthode de Chang et Soong}

Chang et Soong (1979) ont choisi, entre deux possibilités également adéquates pour la loi de distribution du coefficient de consolidation $c_{y}$ (lois log-normales et gamma), la loi gamma. Sur cette base, ils ont déterminé la moyenne et l'écart-type du degré de consolidation résultant de la résolution de l'équation de la consolidation unidimensionnelle d'un mono-couche. Ces coefficients statistiques peuvent s'exprimer en fonction du facteur-temps $T_{*}$ et du coefficient de variation $\mathrm{CV}\left[\mathrm{c}_{\mathrm{v}}\right]$ :

\section{* moyenne}

$$
\begin{aligned}
\bar{U}(t)=1-\sum_{n=0}^{\infty} & \frac{8}{\pi^{2}(2 n+1)^{2}} \times \\
& \times \frac{1}{1+\frac{(2 n+1)^{2} \pi^{2}}{4} \cdot T_{v} \cdot \operatorname{CV}^{2}\left[c_{v}\right]}
\end{aligned}
$$

* variance (carré de l'écart-type)

$$
\begin{aligned}
\sigma_{U}^{2}= & \sum_{n=0}^{\infty} \sum_{m=0}^{\infty} \frac{64}{\pi^{2}(2 n+1)^{2}(2 m+1)^{2}} \times \\
& \times \frac{1}{1+\frac{(2 n+1)^{2}(2 m+1)^{2}}{16} \cdot \pi^{4} \cdot T_{v} \cdot C^{2}\left[c_{v}\right]}
\end{aligned}
$$$$
-(1-\bar{U})^{2}
$$

Le calcul de la moyenne et de l'écart-type du degré de consolidation a été réalisé aux temps $5,10,20$ et 30 ans. Le tableau 4 présente les résultats obtenus dans l'hypothèse où la moyenne et le coefficient de variation de $c_{v}$ valent respectivement

$$
\overline{\mathrm{C}}_{\mathrm{v}}=2,1 \cdot 10^{-8} \mathrm{~m}^{2} / \mathrm{s} ; \mathrm{CV}\left[\mathrm{c}_{\mathrm{v}}\right]=0,76 \text {. }
$$

La figure 6 compare les résultats obtenus par la méthode de Monte-Carlo et par la méthode analytique de Chang et Soong.

$\frac{1}{C V^{2}\left[c_{v}\right]} \quad \frac{1}{C^{2}\left[c_{v}\right]}$

Tableau 4

Résultats du calcul par la méthode de Chang et Soong (1979)

\begin{tabular}{|c|c|c|}
\hline Temps & Moyenne $\bar{U}$ & Écart-type $\sigma_{U}$ \\
\hline 5 ans & 0,48 & 0,03 \\
10 ans & 0,63 & 0,075 \\
20 ans & 0,79 & 0,136 \\
30 ans & 0,86 & 0,174 \\
\hline
\end{tabular}



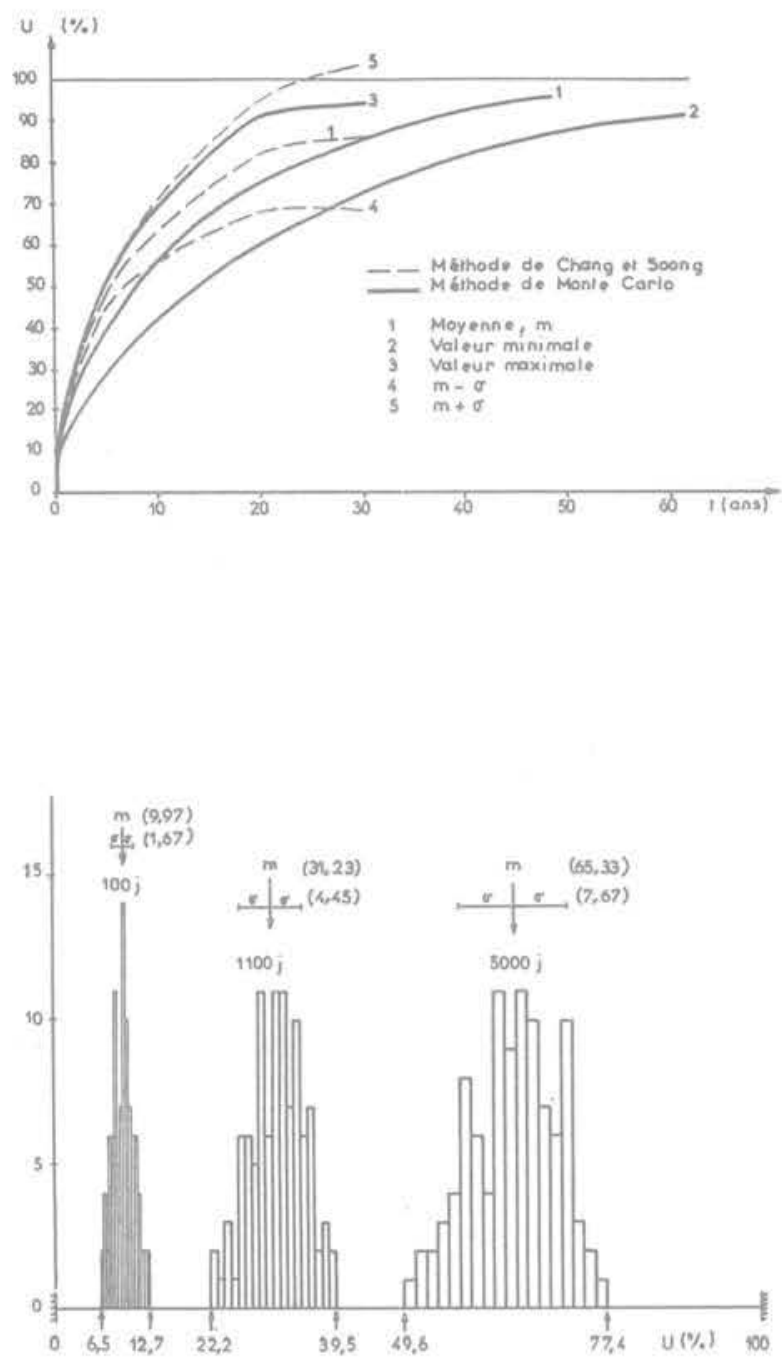

Fig. 7

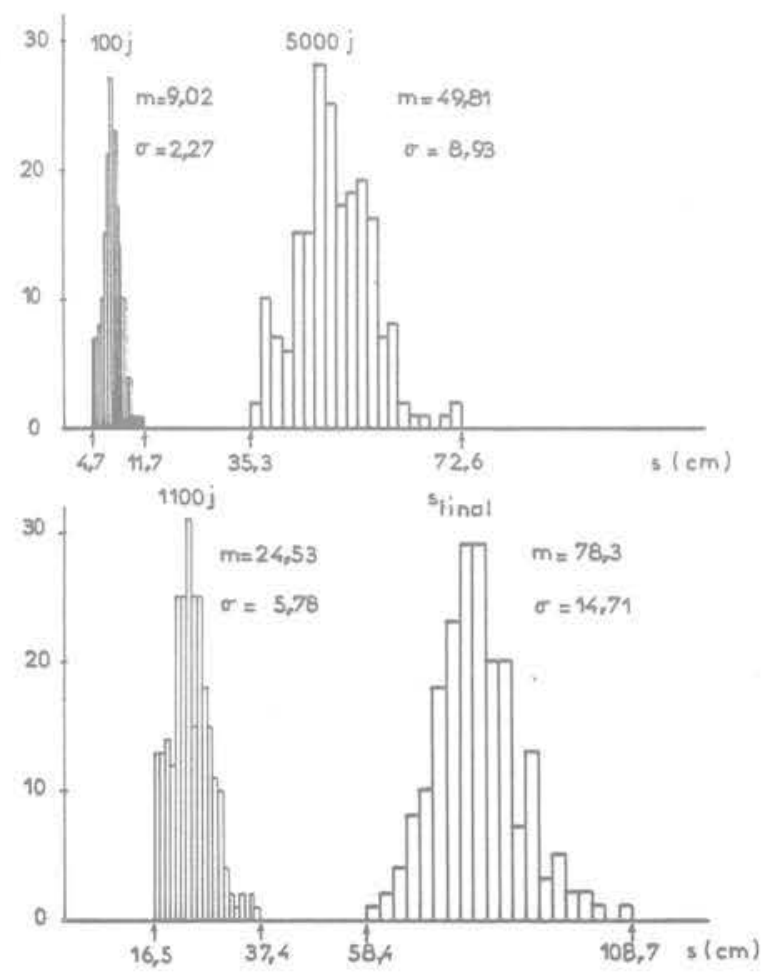

\section{3 Évolution des tassements au cours du temps}

On a utilisé la relation $\mathrm{s}_{\mathrm{t}}=\mathrm{U}(\mathrm{t}) \times \mathrm{s}_{\infty}$ pour calculer le tassement au temps $t$ du sol de fondation du remblai $B$. Dans cette relation, $s_{1}$ et $s_{\infty}$ représentent respectivement le tassement au temps $t$ et le tassement final. Connaissant la distribution et les coefficients statistiques du tassement final $\mathbf{s}_{\infty}$ (fig. 4) et ceux du degré de consolidation $U$ à différents temps ton peut déterminer l'évolution de la distribution du tassement au cours du temps.

Le calcul a été effectué pour $\mathrm{t}=100 \mathrm{j}, 1100 \mathrm{j}$ et $5000 \mathrm{j}$. Pour ces valeurs du temps, on a utilisé la distribution des degrés de consolidation obtenue par la méthode de Monte-Carlo (fig. 5). On a déterminé d'abord la loi de distribution et les coefficients statistiques de chaque $U(t)$. La figure 7 présente les résultats obtenus.

Dans une seconde étape, on a utilisé la méthode de Monte-Carlo pour combiner de façon aléatoire les tassements finaux et les degrés de consolidation: deux cents valeurs de $S_{\infty}$ et $U(t)$ ont été générées pour chaque paramètre puis on a analysé les distributions de $s_{t}=S_{\infty} \cdot U(t)$ aux temps $t=100-1100$ et 5000 jours en calculant leurs coefficients statistiques et en effectuant des tests sur la forme des distributions obtenues. La figure 8 présente la distribution des tassements aux différents temps choisis.

La comparaison des tassements calculés par la méthode indiquée avec les tassements observés au centre du remblai (fig. 9) montre que l'on est loin de prédire de façon correcte le déroulement des tassements au cours du temps. II est très vraisemblable que la différence des comportements calculé et observé provient d'une mauvaise estimation de la valeur réelle du coefficient de consolidation $c_{y}$ en place (la valeur de $c_{v}$ déduite des tassements observés par Magnan et al. (1981) est de $2 \cdot 10^{-7} \mathrm{~m}^{2} / \mathrm{s}$ alors que la valeur moyenne adoptée pour l'étude probabiliste est de 2,1 . $10^{-8} \mathrm{~m}^{2} / \mathrm{s}$ ). Si l'on corrige d'un facteur 10 les temps de tassement probabilistes de la figure 9 , on arrive en effet à faire passer la courbe observée dans le fuseau du calcul, mais cette méthode de correction a posteriori ne peut naturellement pas être utilisée au niveau des projets. En définitive, pour la prévision des vitesses de tassement, l'utilisation des méthodes probabilistes ne peut à elle seule corriger les erreurs traditionnellement commises sur l'ordre de grandeur du coefficient de consolidation.

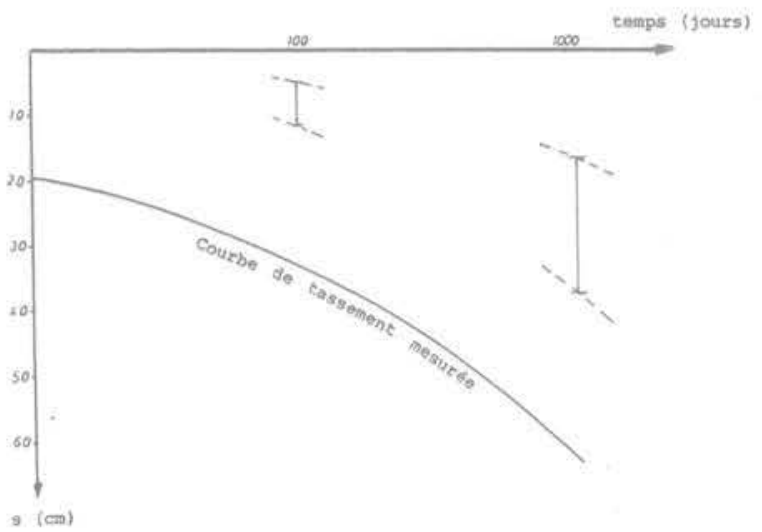

Fig. 9 


\section{Conclusion}

Entreprise dans le but très pragmatique de comparer les différentes méthodes de calcul probabilistes décrites dans la littérature entre elles et avec les résultats des observations faites sur un remblai réel, l'étude décrite dans le présent article a mis en évidence quelques-uns des obstacles à l'utilisation de ce type de méthode au niveau des projets réels et notamment le besoin d'informations statistiques détaillées sur les propriétés des sols (lois de distribution et coefficients de corrélation mais aussi dérive des propriétés avec la profondeur et autocorrélation des valeurs de chaque paramètre). Elle a montré également que les méthodes probabilistes ne peuvent rattraper à elles seules les erreurs commises sur l'ordre de grandeur des paramètres.

Néanmoins, l'approche probabiliste reste prometteuse parce qu'elle doit permettre d'évaluer la marge d'incertitude des résultats des calculs d'ouvrages courants. Des recherches sont engagées dans ce sens, avec notamment pour óbjectif d'estimer les erreurs introduites par la simplification des lois statistiques des propriétés des sols.

\section{Références bibliographiques}

A. Asaoka (1978). Observational procedure of settlement prediction. Soils and Foundations, 18 (4), 87-101.

A. Asaoka, M. Suzuki (1979). Settlement prediction of extensive reclaimed land. Proc. 3rd ICASP, Sydney, 477-486.

A. Belkeziz, J.-P. Magnan (1982). Analyse numérique de la consolidation bidimensionnelle des sols élastoplastiques. Traitement par la méthode deș éléments finis et application au remblai expérimental $B$ de Cubzac-les-Ponts. Laboratoire Central des Ponts et chaussées. Rapport de Recherche LPC. (A paraître.)

J.R. Benjamin, C.A. Cornell (1970). Probability, statistics and decision for civil engineers. Mac-Graw-Hill. New-York.

F. Blondeau, C. Mieussens, D. Queyroi, J.-P. Levillain, M. Peignaud, M. Vogien (1977). Instrumentation du remblai expérimental " $A$ » de Cubzac-les-Ponts. C-R.
International Symposium on Soft Clay, Bangkok. Thaillande, juillet, 419-435.

C.S. Chang, T.T. Soong (1979). A probabilistic approach to consolidation analysis. Proc. 3rd ICASP, Sydney, 487-496.

M.-T. Dang, J.-P. Magnan (1977). Application des modèles élastoplastiques de l'Université de Cambridge au calcul du comportement d'un remblai sur sols mous (site expérimental de Cubzac-les-Ponts). Laboratoire Central des Ponts et chaussées, France, Rapport de Recherche LPC $n^{\circ} 74$.

J.I Folayan, K. Hoeg, J.R. Benjamin (1970). Decision theory applied to settlement predictions. ASCE, Jl of SMFD, Vol. 96, n SM4, July, 1127-1141.

R.A. Freeze (1977), Probabilistic one-dimensional consolidation. ASCE, JI of GED, vol. 103, $n^{\circ}$ GT7, July, 725-742.

J.-P. Magnan, C. Mieussens, D. Queyroi (1978). Comportement du remblai expérimental B à Cubzacles-Ponts. Revue Française de Géotechnique, $n^{\circ} 5$, 23-36.

J.-P. Magnan, S. Baghery, J.-M. Deroy, D. Queyroi (1981). Difficultés du contrôle in situ des calculs de tassements. C.-R. $\mathrm{X}^{\bullet}$ Congrès International de Mécanique des Sols et des Travaux de Fondation. Stockholm. septembre 1981.

S. Shahanguian (1980). Loi de comportement des argiles molles : détermination expérimentale des courbes d'état-limite de l'argile organique de Cubzacles-Ponts. Thèse de docteur-ingénieur. Université Pierre et Marie Curie, Paris, juin 1980.

G. Thomann (1972). Résolution de l'équation de la consolidation unidimensionnelle par la méthode des différences finies. Bull. Liaison Labo. P, et Ch., 59, mai-juin 1972, 57-64.

E.H. Vanmarcke, N.F. Fuleihan (1975). Probabilistic prediction of levee settlement. Proc. 2nd ICASP. Aachen, septembre 1975, 175-190.

M. Vogien (1975). Étude du comportement avant la rupture d'un remblai expérimental construit sur sol mou à Cubzac-les-Ponts. Thèse de docteur-ingénieur. Université Pierre et Marie Curie, Paris. 
